\title{
Estudo piloto sobre possível influência das vitaminas D e B12 nas dislipidemias: alvos para a medicina preventiva
}

\author{
Pilot study about possible influence of vitamins D and B12 values on dyslipidemias: targets \\ for preventive medicine
}
Estudio piloto sobre posible influencia de las vitaminas D y B12 y en las dislipidemias: objetivos de la medicina preventiva

Maíra Reis Pimenta de Queiroz ${ }^{1}$, Isabela Werneck Ranção ${ }^{1}$, Maria Zilda Domingos Abreu Silva ${ }^{1}$, Rodolfo Faria Franco ${ }^{1}$, Kátia Cristina Silva ${ }^{2}$, Nathália Barbosa do Espírito Santo Mendes ${ }^{1}$, Anna Marcella Neves Dias ${ }^{1}$, Danielle Cristina Zimmermann Franco ${ }^{1 *}$.

\section{RESUMO}

Objetivo: Caracterizar uma amostra quanto as dosagens das vitaminas D e B12 e a associação desses valores com os de colesterol total (CT) e frações (HDL-C e LDL-C). Métodos: Estudo transversal, de caráter piloto, realizado a partir de dados coletados em abril/2019 em um laboratório de análises clínicas privado na cidade de Juiz de Fora/MG. Foram incluídos indivíduos $\geq 18$ anos, de ambos os sexos e que fizeram os exames analisados em uma única coleta. Resultados: A amostra $(n=217)$ foi predominantemente formada por mulheres $(71,4 \%)$ e idosos (53,9\%). A prevalência de hipovitaminose $D$ foi de $12,4 \%$ e de B12, $18,5 \%$. Quanto ao HDL-C, apenas 5,53\% apresentavam valores inferiores ao recomendado, já LDL-C e CT, 61,75\% e $57,6 \%$ da amostra, respectivamente, estavam acima dos valores de referência. A partir da avaliação da amostra e corroborando achados de outros estudos sobre a temática, verificou-se correlação forte e moderada, respectivamente, entre os valores de vitamina D e CT e LDL-C. Conclusão: Além de a amostra ter apresentado a necessidade de adequação dos valores de vitamina B12, CT e LDL-C, os valores de vitamina $D$ parecem desempenhar um papel benéfico nas taxas de CT e LDL, podendo ser um alvo importante na Medicina Preventiva.

Palavras-chave: Deficiência de vitaminas, Dislipidemias, Envelhecimento, Assistência integral à saúde.

\begin{abstract}
Objective: To characterize a sample about vitamin D and vitamin B12 levels and their association with values of total cholesterol (TC) and fractions (HDL-C and LDL-C). Methods: Cross-sectional and pilot study carried out from data collect in April/2019, in a private clinical laboratory in Juiz de Fora/MG. Individuals included were age $\geq 18$ years old of both sexes and who made the results in a single collection. Results: The sample $(n=217)$ was composed by women $(71,4 \%)$ and elderly $(53.9 \%)$ in the most part. The prevalence of hypovitaminosis $D$ was $12.4 \%$ and hypovitaminosis B12 was $18.5 \%$. About HDL-C, only $5.53 \%$ had lower values than recommended, whereas LDL-C and CT, $61.75 \%$ and $57.6 \%$ of the sample, respectively, were above the reference values. From the evaluation of the sample and corroborating the findings of other studies on the theme, there was a strong and moderate correlation, respectively, between the values of vitamin $D$ and $C T$ and LDL-C. Conclusion: In addition to the sample showing the need to adjust the values of vitamin B12, CT and LDL-C, vitamin D values seem to play a beneficial role in the rates of CT and LDL. This may be a useful target for Preventive Medicine.
\end{abstract}

Key words: Avitaminosis, Dyslipidemias, Aging, Comprehensive health care.

\section{RESUMEN}

Objetivo: Caracterizar una muestra en relación con las dosis de vitaminas D y B12 y la asociación de estos valores con colesterol total (CT) y factores (HDL-C y LDL-C). Métodos: Estudio transversal, piloto, a partir de

${ }^{1}$ Centro Universitário Presidente Antônio Carlos, Juiz de Fora - MG.

*E-mail: dannyzimmermann@yahoo.com.br

${ }^{2}$ Centro Universitário do Sudeste Mineiro, Juiz de Fora - MG.

SUBMETIDO EM: 4/2021 
datos recogidos en abril/2019 en un laboratorio de análisis clínicos en Juiz de Fora. Fueron incluidos individuos de $\geq 18$ años, de ambos sexos y quién realizó las pruebas analizadas en una sola colección. Resultados: La muestra $(n=217)$ estuvo predominantemente compuesta por mujeres $(71,4 \%)$ y ancianos (53,9\%). El predominio de hipovitaminosis D fue del 12,4\% y B12 del 18,5\%. Sobre el HDL-C, sólo el 5,53\% tuvo valores inferiores a los recomendados, mientras que el LDL-C y el CT, el $61,75 \%$ y el $57,6 \%$ de la muestra, respectivamente, estuvieron por encima de los valores de referencia. Corroborando los hallazgos de otros estudios sobre el tema, se encontró una correlación fuerte y moderada, respectivamente, entre los valores de vitamina D y CT y LDL-C. Conclusión: La muestra indicó la necesidad de ajustar los niveles de vitamina B12, CT y LDL-C. Niveles adecuados de vitamina $D$ parecen desempeñar un papel beneficioso en los valores de CT y LDL. Este puede ser un objetivo útil para la Medicina Preventiva.

Palabras clave: Avitaminosis, Dislipidemias, Envejecimento, Atención integral de salud.

\section{INTRODUÇÃO}

A inadequação e as deficiências de nutrientes essenciais, particularmente em grupos populacionais vulneráveis, adquiriram escala de um problema mundial que merece atenção especial por parte dos profissionais e estudiosos da saúde. Além de implicações de curto prazo para a saúde e qualidade de vida, esse problema pode acarretar consequências de longo prazo, afetando a saúde pública e elevando os custos da superação das complicações associadas às deficiências nutricionais (DARNTON-HILL I, 2019).

Nos últimos anos, diversos estudos foram conduzidos no intuito de explicar a relevância de níveis adequados da vitamina $D(V D)$ e da vitamina $B 12$, bem como seus mecanismos protetores em diferentes patologias (LANGAN RC e ZAWISTOSKI KJ, 2011; PILZ S, et al., 2019). Deve se considerar ainda, que a obesidade, outro problema de saúde pública e fator de risco para inúmeras doenças, incluindo as cardiovasculares (DCV), já foi associada tanto com a deficiência de VD quanto com a de B12 (BALTACI D, et al., 2013; DE AZEVEDO FR e CARAMELI B, 2013).

A VD ou 25 dihidroxi-vitamina $D$ é um nutriente essencial, com atividade semelhante à de um hormônio, que regula o metabolismo do cálcio e dos ossos, além de ser associada ao status imunológico, doenças infecciosas, entre outras (MURDAÇA G, et al., 2019; BRUNO R, et al., 2017). A prevalência de hipovitaminose $D$ é variada pelo globo, embora saiba-se que ela seja elevada em todos os continentes (CACCAMO D, et al., 2018). No Brasil, as prevalências de deficiência e insuficiência de VD foram de $28,16 \%$ e $45,26 \%$, respectivamente (O'CONNOR MY, et al., 2013).

Com relação a vitamina B12 conhecida como cobalamina, apesar de sua reconhecida importância para a síntese de DNA e de energia pelas células, estima-se que $40 \%$ da população na América Latina tenha um quadro clínico ou subclínico de deficiência que pode ser associada, dentre outras, à afecções neurológicas, cutâneas e hematológicas (PEREIRA-SANTOS M, et al., 2019). Em um estudo realizado no estado brasileiro de Minas Gerais, $17,4 \%$ dos participantes apresentavam deficiência dessa vitamina (LANGAN RC e GOODBRED AJ, 2017).

Ambas as vitaminas são alvos constantes de estudos, inclusive sobre a relação das mesmas com as dislipidemias e outros distúrbios metabólicos, relacionando-as, portanto, às DCV (MARTINHO KO, et al., 2015; JIANG X, et al., 2019; DA COSTA REAR, et al., 2020). Sabendo-se que são inúmeras as variáveis que influenciam nos níveis dessas vitaminas, tanto individuais quanto regionais, o conhecimento do perfil de cada população pode ser eficaz no estabelecimento de estratégias para a correção das hipovitaminoses e assim, além de prevenir as manifestações típicas desses quadros, auxiliar na manutenção de níveis saudáveis de colesterol total (CT) e frações de baixa densidade (LDL-C) e alta densidade (HDL-C) (ADAIKALAKOTESWARI A, et al., 2015; PORTO CM, et al., 2019).

Desse modo, o presente estudo, com caráter piloto, teve por objetivo, primeiramente, verificar a situação de saúde (status nutricional) de usuários de um serviço privado de Análises Clínicas no município de Juiz de Fora/MG de acordo com as dosagens de vitaminas D, B12, CT e frações. Em seguida, investigar a associação entre os valores de VD e vitamina B12 com os valores de CT e frações encontrados, a fim de determinar se a carência destes nutrientes (VD e cobalamina) pode se relacionar, na prática clínica, às dislipidemias em tal 
população, sugerindo que a manutenção de níveis desejáveis dessas vitaminas sejam importantes para a prevenção de doenças crônicas que afetam a população de todo mundo.

\section{MÉTODOS}

O estudo foi realizado a partir dos dados de usuários de um laboratório de Análises Clínicas privado. 0 serviço situa-se no município de Juiz de Fora, Minas Gerais que conta com população de 570 mil habitantes.

Tratou-se de um estudo transversal, retrospectivo, com caráter piloto, a partir da análise de componentes descritivos e analíticos, baseado na coleta de dados de pacientes que enquadraram-se nos seguintes critérios de inclusão: ambos os sexos, com idade igual ou superior a 18 anos, que realizaram, de acordo com solicitação médica, dosagens referentes às vitaminas $D$ (a partir do intermediário 25(OH)VD) e B12, bem como dos parâmetros bioquímicos colesterol total (CT) e frações (LDL-C e HDL-C), numa mesma coleta, em 2019, no laboratório escolhido. Foram excluídos pacientes dos quais pelo menos uma das dosagens foi incerta ou corrompida.

O cálculo amostral foi feito com base no total de atendimentos realizados entre janeiro e abril de 2019 , totalizando 900 indivíduos. A partir daí, utilizou-se a fórmula para cálculo amostral de populações finitas com nível de confiança de $90 \%$, quantidade de acerto esperado de $90 \%$ e nível de precisão de $3 \%$. Assim, 217 indivíduos foram incluídos, sendo possível selecionar todos os exames de um mesmo mês (abril/2019), minimizando as variações sazonais na exposição solar que poderia afetar a vitamina $D$ dos participantes.

Para classificação dos níveis de VD, considerou-se como hipovitaminose os valores abaixo de $20 \mathrm{ng} / \mathrm{mL}$ do intermediário $25(\mathrm{OH})$ vitamina $\mathrm{D}$. Já para vitamina B12, foram considerados como insuficientes os valores inferiores a $150 \mathrm{pg} / \mathrm{mL}$ (LANGAN RC e GOODBRED AJ, 2017). Para o colesterol e frações, adotou-se para fins de avaliação da amostra os seguintes valores de referência (VR): $\mathrm{CT}<190 \mathrm{mg} / \mathrm{dL}$ (desejável); HDL-C $>40$ $\mathrm{mg} / \mathrm{dL}$ (desejável) e LDL-C $<100 \mathrm{mg} / \mathrm{dL}$ (referente a categoria de risco intermediário) (FALLUDI AA, et al., 2017).

Os resultados das dosagens foram analisados de acordo com sexo e idade aplicando-se estatística descritiva e a comparação das médias \pm desvio padrão foi feita pelo teste paramétrico (Teste t) e ANOVA seguido pelo teste de Tukey $(p<0,05)$. O teste de normalidade aplicado foi o de Shapiro-Wilk. Para análise de correlação entre os valores séricos das vitaminas $D$ e B12 e valores de $C T$ e frações, foi realizado o teste de correlação de Spearman para as variáveis não-paramétricas e de Fisher, para aquelas paramétricas $(p<0,05)$. Para avaliação do valor de $r$, foram adotados os seguintes parâmetros: $r<0,40=$ fraca; $0,40<r<0,69=$ moderada e $r>0,70$, forte (SIQUEIRA AL e TIBÚRCIO JD, 2011). Em estudos clínicos e biomédicos, a maior parte dos coeficientes com significado biológico situa-se entre 0,5 e 0,8 (ou -0,5 e -0,8) (MIOT HA, 2018).

A análise e armazenamento dos dados foram realizadas nos softwares Excel e Graph Pad Prism 6 e os resultados, expressos em tabelas. Este estudo foi aprovado pelo Comitê de Ética em Pesquisa com Seres Humanos do Centro Universitário Presidente Antônio Carlos, Barbacena - MG (Número do parecer: 3.560.715).

\section{RESULTADOS}

A amostra $(n=217)$ apresentou média de idade de 52,06 $\pm 18,07$ anos e predominância do sexo feminino $(n=155,71,4 \%)$. A média de idade por sexo demonstrou que as mulheres tinham $52,44 \pm 18,18$ anos e os homens $51,09 \pm 17,88$ anos ( $p>0,05)$. Além disso, foi possível identificar que $53,9 \%(n=117)$ da amostra eram idosos ( $\geq 60$ anos) (Tabela 1).

No que diz respeito ao HDL-C, a amostra apresentou valor médio de $60,85 \pm 14,73 \mathrm{mg} / \mathrm{dL}$. Foram constatados apenas 12 (5,53\%) indivíduos com HDL-C inferior ao recomendado. Avaliando segundo o sexo, homens e mulheres apresentaram médias que atendiam ao valor de referência para esse parâmetro e os valores não foram estatisticamente distintos entre eles $(p=0,7452)$ (Tabela 2). 
Tabela 1 - Caracterização dos pacientes atendidos pelo laboratório privado de análises clínicas e selecionados para o presente estudo, de acordo com a faixa etária e sexo. $n=217$. Juiz de Fora MG, 2021.

\begin{tabular}{|c|c|c|}
\hline Faixa etária (anos) & $\mathbf{N}$ & $\%$ \\
\hline $18-29$ & $\begin{array}{c}31 \\
8 \text { homens e } 23 \text { mulheres }\end{array}$ & $\begin{array}{c}14,28 \% \\
3,68 \% \text { homens e } 10,59 \% \text { mulheres }\end{array}$ \\
\hline $30-39$ & $\begin{array}{c}33 \\
11 \text { homens e } 22 \text { mulheres }\end{array}$ & $\begin{array}{l}15,20 \% \\
5,06 \% \text { homens e } 10,12 \% \text { mulheres }\end{array}$ \\
\hline $40-50$ & $\begin{array}{c}36 \\
12 \text { homens e } 24 \text { mulheres }\end{array}$ & $\begin{array}{c}16,58 \% \\
5,52 \% \text { homens e } 11,05 \% \text { mulheres }\end{array}$ \\
\hline $51-60$ & $\begin{array}{c}44 \\
15 \text { homens e } 29 \text { mulheres }\end{array}$ & $\begin{array}{c}20,27 \% \\
6,91 \% \text { homens e } 13,36 \% \text { mulheres }\end{array}$ \\
\hline $61-70$ & $\begin{array}{c}45 \\
11 \text { homens e } 34 \text { mulheres }\end{array}$ & $\begin{array}{c}20,73 \% \\
5,06 \% \text { homens e } 15,66 \% \text { mulheres }\end{array}$ \\
\hline $71-80$ & 18 mulheres e 7 homens & $\begin{array}{c}11,52 \% \\
8,29 \% \text { homens e } 3,22 \% \text { mulheres }\end{array}$ \\
\hline $81-95$ & $\begin{array}{c}3 \\
0 \text { homens e } 3 \text { mulheres }\end{array}$ & $\begin{array}{c}1,38 \% \\
0 \% \text { homens e } 1,38 \% \text { mulheres }\end{array}$ \\
\hline
\end{tabular}

Fonte: Queiroz MRP, et al., 2021.

Ao analisar o valor de LDL-C, a média geral da população foi de $113,89 \pm 42,05 \mathrm{mg} / \mathrm{dL}$. Verificou-se que $134(61,75 \%)$ indivíduos apresentavam valor acima do VR e que as faixas etárias entre 51 e 60 anos e 61 e 70 anos ( $n=31,14,3 \%$ em ambas, totalizando 62 pacientes, 28,6\%) demonstraram a maior frequência de indivíduos fora do VR. Segundo o gênero, mulheres apresentaram LDL-C médio menor do que dos homens $(111,4 \pm 38,02 \mathrm{mg} / \mathrm{dL}$ e $120,1 \pm 50,42 \mathrm{mg} / \mathrm{dL}$, respectivamente, $\mathrm{p}=0,0061)$, embora ambos tenham apresentado valores acima da referência (Tabela 2).

Com relação ao CT, a média geral da população correspondeu a 196,68 $\pm 41,80 \mathrm{mg} / \mathrm{dL}$ extrapolando, mais uma vez, o valor de referência. Dentre os analisados, 125 (57,6\%) estavam acima do valor estabelecido como desejável e a faixa etária de 61 a 70 anos foi aquela com maior número de indivíduos acima desse valor $(n=31,14,3 \%)$. Ponderando segundo o gênero, mulheres $(226,0 \pm 40,08 \mathrm{mg} / \mathrm{dL})$ e homens $(195,8 \pm 46,30$ $\mathrm{mg} / \mathrm{dL})$, novamente, se enquadraram fora do valor de referência $(<190 \mathrm{mg} / \mathrm{dL})($ Tabela 2$)$.

Tabela 2 - Valores médios de CT e frações segundo as variáveis faixa etária e sexo dos pacientes atendidos pelo laboratório privado de análises clínicas em Juiz de Fora-MG e selecionados para o presente estudo. n=217. Juiz de Fora - MG, 2021.

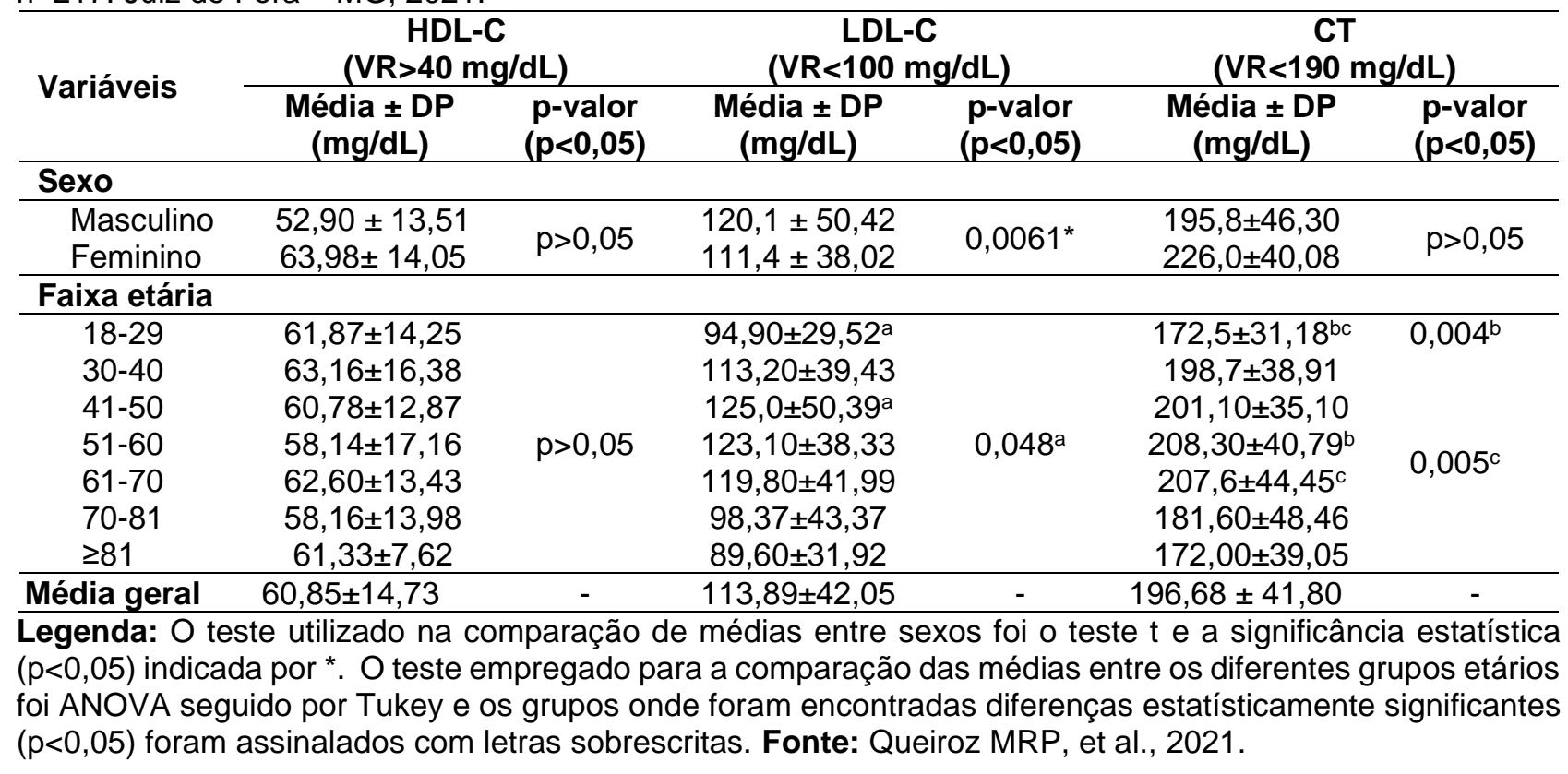


A avaliação dos níveis de vitamina B12 da amostra revelou média de 280,86 $\pm 133,03 \mathrm{pg} / \mathrm{mL}$ e adequação ao VR por todas as faixas etárias. Observou-se dois casos de hipervitaminose no grupo de 74 a 84 anos, com ambos os exemplares superiores a $1.500 \mathrm{pg} / \mathrm{mL}$, valor máximo detectável pelo método utilizado no diagnóstico. Não houve diferença nos valores de acordo com o gênero ( $p=0,2907)$. Foram identificados 40 $(18,5 \%)$ casos de hipovitaminose (Tabela 3).

Por fim, a avaliação dos níveis de $25(\mathrm{OH})$ Vitamina D revelou média de $29,31 \pm 9,05 \mathrm{ng} / \mathrm{mL}$, tendo o sexo feminino valores mais baixos do que o masculino $(29,05 \pm 8,46 \mathrm{ng} / \mathrm{mL}$ e $30,00 \pm 10,46 \mathrm{ng} / \mathrm{mL}$, respectivamente. $\mathrm{p}=0,0397$ ). Não foram encontradas diferenças entre as faixas etárias e todas se encontravam acima do VR. Identificou-se 27 (12,4\%) indivíduos em situação de hipovitaminose D (Tabela 3).

Tabela 3 - Valores médios das vitaminas D e B12, segundo as variáveis faixa etária e sexo dos pacientes atendidos pelo laboratório privado de análises clínicas em Juiz de Fora-MG e selecionados para o presente estudo. $n=217$. Juiz de Fora - MG, 2021.

\begin{tabular}{|c|c|c|c|c|}
\hline \multirow{2}{*}{ Variáveis } & \multicolumn{2}{|c|}{$\begin{array}{c}\text { 25(OH)Vit D } \\
(\mathrm{VR}>20 \mathrm{ng} / \mathrm{mL}) \\
\end{array}$} & \multicolumn{2}{|c|}{$\begin{array}{c}\text { Vitamina B12 } \\
(\text { VR>150 } \mathrm{pg} / \mathrm{mL})\end{array}$} \\
\hline & $\begin{array}{l}\text { Média } \pm \text { DP } \\
(\mathrm{ng} / \mathrm{mL})\end{array}$ & $\begin{array}{c}p \text {-valor } \\
(p<0,05)\end{array}$ & $\begin{array}{l}\text { Média } \pm \text { DP } \\
(\mathrm{pg} / \mathrm{mL})\end{array}$ & $p$-valor $(p<0,05)$ \\
\hline \multicolumn{5}{|l|}{ Sexo } \\
\hline $\begin{array}{l}\text { Masculino } \\
\text { Feminino } \\
\end{array}$ & $\begin{array}{c}30,00 \pm 10,46 \\
29,05 \pm 8,46\end{array}$ & $0,0397^{*}$ & $\begin{array}{l}288,9 \pm 144,7 \\
279,4 \pm 129,4 \\
\end{array}$ & $p>0,05$ \\
\hline \multicolumn{5}{|l|}{ Faixa etária } \\
\hline $18-29$ & $28,63 \pm 9,683$ & & $254,1 \pm 84,11$ & \\
\hline $30-40$ & $30,6 \pm 7,595$ & & $285,9 \pm 105,9$ & \\
\hline $41-50$ & $27,89 \pm 8,642$ & & $321,5 \pm 167,4$ & \\
\hline $51-60$ & $29,39 \pm 7,837$ & $p>0,05$ & $313,3 \pm 151,2$ & $p>0,05$ \\
\hline $61-70$ & $29,59 \pm 11$ & & $253,5 \pm 108,1$ & \\
\hline $71-80$ & $28,64 \pm 7,537$ & & $335,6 \pm 379,5$ & \\
\hline$\geq 81$ & $40,37 \pm 16,57$ & & $307,7 \pm 63,54$ & \\
\hline Média geral & $29,31 \pm 9,05$ & - & $288,9 \pm 144,7$ & $p>0,05$ \\
\hline
\end{tabular}

Legenda: $O$ teste utilizado na comparação de médias entre sexos foi $o$ teste $t$ e a significância estatística $(p<0,05)$ indicada por *. O teste empregado para a comparação das médias entre os diferentes grupos etários foi ANOVA seguido por Tukey, mas não foi encontrada diferença entre elas.

Fonte: Queiroz MRP, et al., 2021.

Por fim, foi feita a análise de correlação entre os valores encontrados de vitaminas $D$ e $B 12$ e os valores de CT, LDL-C e HDL-C. Dessa forma, foram verificadas as seguintes forças de correlação: forte entre 25(OH)Vit D e CT; e moderada entre 25(OH)Vit D e LDL, sendo em ambos os casos percebido que o aumento de VD acompanha a redução de CT e LDL. No caso do HDL, foi percebido que o aumento de VD acompanhava o aumento de HDL, porém a correlação foi fraca. Já no caso da vitamina B12, todas as correlações foram fracas, sendo a de HDL-C sem significância estatística.

Tabela 4 - Correlação entre os valores de vitaminas $D$ e B12 na amostra pesquisada e os valores de Colesterol Total (CT), LDL-C e HDL-C. n=217. Juiz de Fora - MG, 2021.

\begin{tabular}{|c|c|c|c|c|c|c|}
\hline \multirow{2}{*}{ Valores } & \multirow{2}{*}{$r$} & \multicolumn{2}{|c|}{$\begin{array}{c}25(\mathrm{OH}) \text { Vit D } \\
(\mathrm{VR}>20 \mathrm{ng} / \mathrm{mL})\end{array}$} & \multicolumn{3}{|c|}{$\begin{array}{c}\text { Vitamina B12 } \\
\text { (VR>150 pg/mL) }\end{array}$} \\
\hline & & IC 95\% & $\begin{array}{c}\text { p-valor } \\
(p<0,05)\end{array}$ & $r$ & IC 95\% & $\begin{array}{c}\mathrm{p} \text {-valor } \\
(\mathrm{p}<0,05)\end{array}$ \\
\hline HDL-C & 0,2009 & $0,06528-0,3292$ & $0,0030^{*}$ & 0,0108 & $-0,1274-0,1486$ & 0,8755 \\
\hline LDL-C & $-0,6536$ & $-0,7122-0,5859$ & $<0,0001^{*}$ & 0,1607 & $0,0517-0,2660$ & $0,0031^{*}$ \\
\hline CT & $-0,7541$ & $-0,7978-0,7025$ & $<0,0001^{*}$ & 0,1384 & $0,0289-0,2446$ & $0,0110^{*}$ \\
\hline
\end{tabular}

Legenda: Para HDL-C foi aplicado o teste de correlação de Fisher. Para LDL-C e CT foi aplicado o teste de correlação de Spearman. * Valor estatisticamente significante $(p<0,05)$. Fonte: Queiroz MRP, et al., 2021. 


\section{DISCUSSÃO}

A situação de hipovitaminose e insuficiência das vitaminas D e B12 tem sido amplamente mencionada e discutida na literatura (LANGAN RC e ZAWISTOSKI KJ, 2011; PILZ S, et al., 2019). A realização desse estudo piloto revelou que a amostra em análise se demonstrou com necessidade de adequação quanto aos valores de LDL-C e CT, e possibilidade de elevação nos níveis de ambas as vitaminas para se obter benefícios específicos, especialmente por se tratar de indivíduos idosos ou com idade próxima aos 60 anos.

Essa investigação permitiu a observação de que a concentração de $25(\mathrm{OH})$ vitamina $D$ foi inversamente correlacionado com o valor de CT e LDL-C; e positivamente correlacionado com o valor de HDL-C. Esses achados corroboram estudos que indicaram um risco aumentado de mortalidade por DCV quando os níveis circulantes de 25(OH) vitamina D estão abaixo de 25 nmol/L (JIANG X, 2019; ZITTERMANN A, et al., 2011).

A deficiência de vitamina B12 também já foi relacionada como um importante fator de risco metabólico. Na situação de hipovitaminose, algumas regiões de genes envolvidos na síntese do colesterol tornam-se hipometiladas e esse pode ser um mecanismo importante pelo qual há um aumento na via de biossíntese do colesterol nos adipócitos humanos (ADAIKALAKOTESWARI A, et al., 2015; BOACHIE J, et al., 2020).

Outra questão importante com relação a deficiência da vitamina B12 é que tal situação contribui para o aumento do risco de DCV por meio de sua associação com o nível de homocisteína (tHcy), uma vez que na via de remetilação da homocisteína haverá uma diminuição da metionina sintase, que é dependente de vitamina B12 e que converte homocisteína em metionina (MCLEAN RR e HANNAN MT, 2007; COUSSIRAT $C$, et al., 2012). Ademais, a suplementação com tal vitamina foi associada a uma redução de aproximadamente 7\% no nível de tHcy (BAZANO LA, et al., 2006).

No presente estudo, verificou-se que, avaliando quanto a idade e gênero, houve adequação da amostra aos valores de referência propostos para VD e vitamina B12. Contudo, esses valores mínimos tidos como referência (20 ng/mL para VD e de $150 \mathrm{pg} / \mathrm{mL}$, para Vitamina B12) ainda são controversos e questionados se suficientes para se obter todos os benefícios mediados por elas.

Em uma meta-análise realizada por Wang L, et al. (2012) baseada nos resultados de 16 estudos, a associação dose-resposta entre a vitamina $25(\mathrm{OH})$ vitamina $\mathrm{D}$ circulante e o risco de DCV foi inversamente proporcional, demostrando que o risco das mesmas aumenta com a diminuição da concentração de VD abaixo de $60 \mathrm{nmol} / \mathrm{L}$. Isso ocorre devido ao fato da vitamina $D$ atuar inibindo a expressão gênica da renina, levando à diminuição da sua síntese (YUAN W, et al., 2007; LAGOEIRO JAJ, et al., 2018).

De acordo com Langan RC e Goodbred AJ (2017), valores entre 30 e $60 \mathrm{ng} / \mathrm{mL}$ seriam recomendados para grupos de risco como idosos, gestantes, pacientes com osteomalácia, raquitismos, osteoporose, hiperparatireoidismo secundário, doenças inflamatórias, doenças autoimunes e renal crônica e prébariátricos. Considerando esse valor mais elevado, a amostra estaria inadequada no que tange à VD. Contudo, comparando a frequência de hipovitaminose aqui encontrada $(12,4 \%)$ com a da população brasileira (28,16\%) (PEREIRA-SANTOS M, et al., 2019). A amostra apresentou melhor condição. Embora não tenha sido realizada a investigação de dados socioeconômicos da população em estudo, o fato de se tratar de usuários de um Laboratório de Análises Clínicas privado pode ter relação com um melhor acesso a cuidados de saúde.

Sob essa mesma perspectiva, para a vitamina B12, já foi sugerido que o nível adequado, especialmente para idosos, deva ser igual ou maior que $250 \mathrm{pg} / \mathrm{mL}$ (KAKEHASI AM, et al., 2012). Nesse estudo, mesmo considerando valores mais elevados para B12, a situação da amostra foi satisfatória. No entanto, foi perceptível a diferença entre os valores aqui demonstrados e os relatados em outro estudo semelhante: em uma amostra composta por mulheres menopausadas com idade média de 62,5 $\pm 6,9$ anos, os valores de vitamina B12 encontrados foram de $565 \pm 439 \mathrm{pg} / \mathrm{mL}$ (KAKEHASI AM, et al., 2012). Comparativamente a esses dados, a amostra analisada apresentou níveis séricos de vitamina B12 cerca de duas vezes menores.

Cabe ressaltar que essa investigação teve caráter piloto e uma das limitações encontradas deveu-se a impossibilidade de se avaliar a presença de fatores de risco que contribuíram para valores mais baixos de 
ambas as vitaminas nessa amostra, tais como cor da pele e tempo de exposição solar diário para VD; e doenças no trato gastrointestinal, hábitos alimentares e realização de cirurgia bariátrica, para B12, que podem ter influenciado nos valores médios dessa população de modo desconhecido (FERREIRA-NUNES PM, et al., 2018; LANGAN RC e GOODBRED AJ, 2017). O único fator de risco para as hipovitaminoses que pode ser afirmado como presente na amostra é o da idade, já que houve predomínio de idosos (53,9\%), já tendo sido relatado que o envelhecimento é associado a níveis mais baixos dessas vitaminas (FERREIRA-NUNES PM, et al., 2018).

Com relação aos valores de CT e frações, a amostra em análise demonstrou uma melhor condição para HDL-C quando comparada a situação nacional: em um estudo realizado sobre o perfil lipídico no Brasil, a média populacional em relação ao HDL-C foi de $46,5 \mathrm{mg} / \mathrm{dL}$, sendo de $43 \mathrm{mg} / \mathrm{dL}$ para o sexo masculino e de 49,6 mg/dL para o feminino (MALTA DC, et al., 2019). Além de atender a recomendação para o valor mínimo de HDL-C, já foi sugerido que este também não seja superior a $97 \mathrm{mg} / \mathrm{dL}$ para homens e $135 \mathrm{mg} / \mathrm{dL}$ para mulheres, uma vez que valores tão elevados de HDL foram associados, paradoxalmente, a um maior risco cardiovascular (SINGH K e ROHATGI A, 2018). Na presente análise não foram encontrados valores superiores a essa recomendação.

Foram, no entanto, identificadas inadequações na amostra referentes aos valores de referência de LDL-C e CT, semelhantes ao reportado como sendo a situação brasileira (MALTA DC, et al., 2019). Assim, a faixa etária com valores mais elevados de LDL-C e CT, em ambos os casos, estavam compreendidos no intervalo de 40 a 59 anos e, para CT, identificou-se que a média geral da amostra analisada foi superior à média brasileira, bem como o valor médio para o sexo feminino na amostra, superior à média brasileira.

Observou-se, por fim, correlação entre os valores de 25(OH)VD e os de CT e LDL-C de forma semelhante ao reportado por estudos recentes como o de Dibaba DT (2019), que indicaram que a VD seria capaz de ter um efeito benéfico na redução de CT e LDL-C; e de AIQuaiz AM, et al. (2020) ao demonstrarem que, na população saudita, a VD influenciou o perfil lipídico sérico e que a manutenção de um nível sérico satisfatório dessa vitamina pode resultar em níveis lipídicos favoráveis.

Embora outros fatores de risco para dislipidemias e DCV pudessem estar presentes na amostra, o fator protetor oferecido pela VD não deve ser ignorado. No que tange a vitamina B12, acreditou-se que a sua deficiência tenha que ser ainda mais acentuada e persistente para afetar os mecanismos de biossíntese do colesterol. No presente estudo, $81,5 \%$ dos participantes apresentavam níveis baixos, mas não insuficientes, segundo valores propostos por Langan RC e Goodbred AJ (2017).

Os achados relacionados aos níveis baixos da vitamina B12, devem ser avaliados ao mesmo tempo que se busca comparações da mesma natureza na literatura e em populações maiores, controlando-se também todas as possíveis fontes de interferência nessa observação. Em análises de correlação, é preciso ter em foco que fenômenos biológicos sofrem influências multifatoriais e interações complexas, em que a variação de apenas uma variável não explica totalmente o comportamento de outra (MIOT H, 2018).

Com base nos achados, o grupo analisado demonstrou-se oportuno para ajustes dos níveis vitamínicos por modificações no estilo de vida e/ou pelo uso de suplementação vitamínica, o que é de particular interesse para a Medicina Preventiva. Reestabelecer valores adequados de vitamina $D$ pode ser uma parte importante no tratamento e prevenção das dislipidemias a partir, especialmente, em idosos. O objetivo dessa adequação abrange a prevenção das manifestações diretamente relacionadas às hipovitaminoses, especialmente agravadas pelo envelhecimento. Além disso, a VD tem demonstrado ser um recurso que pode ser implementado na prática clínica médica para auxiliar na adequação e manutenção de níveis satisfatórios de CT e LDL-C, necessidade esta que despontou como iminente para a amostra e já foi relatada, em outros estudos, em diferentes regiões brasileiras.

\section{CONCLUSÃO}

Concluiu-se que os valores de vitamina $D$ apresentaram correlação forte e moderada, respectivamente, com os valores de CT e LDL-C na amostra em questão. Não foi encontrada correlação significativa entre 
valores inadequados de vitamina B12 e as dislipidemias. Como esse estudo tratou-se de um piloto para delineamento da situação da amostra e da busca por uma relação entre as hipovitaminoses e dislipidemias, investigações mais específicas na população em questão deverão ser realizadas de modo a considerar todos os fatores que podem influenciar nessa observação.

\section{REFERÊNCIAS}

1. ADAIKALAKOTESWARI $A$, et al. Vitamin B12 insufficiency induces cholesterol biosynthesis by limiting $s-$ adenosylmethionine and modulating the methylation of SREBF1 and LDL-CR genes. Clin Epigenetics, 2015; 7(1): 114.

2. ALQUAIZ AM, et al. Association between standardized vitamin 25(OH)D and dyslipidemia: a community-based study in Riyadh, Saudi Arabia. Environ Health Prev Med, 2020; 25(1): 1-9.

3. BALTACI D, et al. Association of vitamin B12 with obesity, overweight, insulin resistance and metabolic syndrome, and body fat composition; primary care-based study. Med Glas (Zenica), 2013; 10(2): 203-10.

4. BAZZANO LA, et al. Effect of folic acid supplementation on risk of cardiovascular diseases: a meta-analysis of randomized controlled trials. JAMA, 2006; 296: 2720-6.

5. BRUNO R, et al. Prevalence of micronutrients deficiencies in a cohort of HIV-positive individuals on ART. Infect Dis Trop Med, 2017; 3: 1-4.

6. CACCAMO D et al. Health Risks of Hypovitaminosis D: A Review of New Molecular Insights. Int J Mol Sci. 2018; 19(3): 1-18.

7. COUSSIRAT C, et al. Vitaminas B12, B6, B9 e homocisteína e sua relação com a massa óssea em idosos. Rev. Bras Geriatr Gerontol, 2012; 15(3): 577-85.

8. DA COSTA REAR, et al. Deficiência de Vitamina D no Diabetes Mellitus: revisão integrativa da literatura. Revista Eletrônica Acervo Saúde, 2020; (57): e3994.

9. DARNTON-HILL I. Public Health Aspects in the Prevention and Control of Vitamin Deficiencies. Curr Dev Nutr, 2019; 3(9): 1-14.

10. DE AZEVEDO FR, CARAMELLI B. Hypovitaminosis D and Obesity - Coincidence or Consequence? Eur Endocrinol, 2013; 9(2): 128-31.

11. DIBABA DT. Effect of vitamin D supplementation on serum lipid profiles: a systematic review and meta-analysis. Nutr Rev, 2019; 77(12): 890-902.

12. FALLUDI AA, et al. Atualização da diretriz brasileira de dislipidemias e prevenção da aterosclerose - 2017. Arquivos Brasileiros de Cardiologia. 2017; 109(1): 1-76.

13. FERREIRA-NUNES PM, et al. Padrões alimentares e ingestão de nutrientes em idosos: análise com diferentes abordagens metodológicas. Ciênc Saúde Coletiva, 2018; 23(12): 4085-94.

14. BOACHIE J, et al. Low Vitamin B12 and Lipid Metabolism: Evidence from Pre-Clinical and Clinical Studies. Nutrients. 2020; 12(7): 1925.

15. JIANG X, et al. Vitamin D deficiency is associated with dyslipidemia: a cross-sectional study in 3788 subjects. Curr Med Res Opin, 2019; 35(6): 1059-63.

16. KAKEHASI AM, et al. Níveis séricos de vitamina B12 não se relacionam com baixa densidade mineral óssea em mulheres brasileiras na pós-menopausa. Rev Bras Reumatol, 2012; 52(6): 863-9.

17. LAGOEIRO JAJ, et al. Vitamin D Deficiency and Cardiovascular Diseases. Int J Cardiovasc Sci, 2018; 31(4): 422-32.

18. LANGAN RC, GOODBRED AJ. Vitamin B12 Deficiency: Recognition and Management. Am Fam Physician, 2017; 96(6): 384-9.

19. LANGAN RC, ZAWISTOSKI KJ. Update on vitamin B12 deficiency. Am Fam Physician. 2011; 83(12): $1425-30$.

20. MALTA DC, et al. Prevalência de colesterol total e frações alterados na população adulta brasileira: Pesquisa Nacional de Saúde. Rev Bras Epidemiol, 2019; 22(Suppl 2): 1-13.

21. MARTINHO KO, et al. Prevalence and factors associated with vitamin b12 deficiency in elderly from Viçosa/MG, Brazil Nutr Hosp, 2015; 32(5): 2162-8.

22. MCLEAN RR, HANNAN MT. B vitamins, homocysteine, and bone disease: epidemiology and pathophysiology. Curr Osteoporos Rep, 2007; 5(3): 112-9.

23. MIOT HA. Análise de correlação em estudos clínicos e experimentais. J Vasc Bras, 2018; 17(4): 275-279.

24. MURDACA G, et al. Emerging role of vitamin $D$ in autoimmune diseases: An update on evidence and therapeutic implications. Autoimmun Rev, 2019; 18(9): 1-10.

25. O'CONNOR MY, et al. The uncertain significance of low vitamin D levels in African descent populations: a review of the bone and cardiometabolic literature. Prog Cardiovasc Dis, 2013; 56(3): 261-9. 
26. PEREIRA-SANTOS M, et al. Epidemiology of vitamin D insufficiency and deficiency in a population in a sunny country: Geospatial meta-analysis in Brazil. Crit Rev Food Sci Nutr, 2019; 59(13): 2102-9.

27. PILZ S, et al. Vitamin D testing and treatment: a narrative review of current evidence. Endocr Connect, 2019; 8(2): R27-R43.

28. PORTO CM, et al. Contribuições da vitamina D no tratamento de sintomas depressivos e fatores de risco cardiovascular: protocolo de estudo para um ensaio clínico randomizado, duplo-cego e controlado por placebo. Trials, 2019; 20(1): 1-9.

29. SINGH K, ROHATGI A. Examining the paradox of high high-density lipoprotein and elevated cardiovascular risk. J Thorac Dis, 2018; 10(1): 109-12.

30. SIQUEIRA AL, TIBÚRCIO JD.Estatística na área de saúde: conceitos, metodologia, aplicações e prática computacional. Belo Horizonte: Coopmed; 2011; 520p

31. WANG L, et al. Circulating 25-hydroxy-vitamin D and risk of cardiovascular disease: a meta-analysis of prospective studies. Circ Cardiovasc Qual Outcomes, 2012; 5(6): 819-29.

32. YUAN W, et al. 1,25-dihydroxyvitamin D3 suppresses renin gene transcription by blocking the activity of the cyclic AMP response element in the renin gene promoter. J Biol Chem, 2007; 282(41): 29821-30.

33. ZITTERMANN A, et al. The role of vitamin D in dyslipidemia and cardiovascular disease. Curr Pharm Des, 2011; 17(9): 933-42. 